can have. The aim of the 'Changing attitudes and behaviours towards digital legacy and digital assets planning' poster will be to display this year's data. We will do so by cross-referencing this year's data with data from the previous two years.

Methods Cross reference data, explore year on year changes, draw conclusions and predict trends. We launched the Digital Death Survey at EAPC 2016. The survey coincided with the first interactive poster that displays real time data on it.

Results 300 people have completed the survey thus far. We expect 500 people to have completed it by the end of July when the survey ends. We plan to display the data digitally (via an iPad) on the poster and through images on the poster.

\section{P-31 SAFEGUARDING FOR A DIGITAL SERVICE: THE SUE RYDER ONLINE COMMUNITY}

Priscilla McClay, Jacqui Graves, Sue Hogston. Sue Ryder, London, UK

\subsection{6/bmjspcare-2017-hospice.58}

Background The Sue Ryder Online Community is an online peer support service where people affected by terminal illness or bereavement exchange messages and support each other. The site is moderated to keep it safe and supportive. Because of the difficult subject matter, individuals may occasionally post about suicidal thoughts and feelings, and it is necessary to have a robust process for responding.

Aims To ensure that individuals considered at risk are supported and signposted to appropriate services, and that our approach is consistent.

Methods A decision flow chart helps moderators assess the level of risk and gives an easy-to-follow process. Template responses and lists of signposting resources are used by moderators to support individuals and encourage them to seek specialist help - these are adapted and personalised in each case. Issues where an individual is considered at-risk are escalated to senior health and social care staff. Following assessment, in a very high-risk case, we consider, on a risk-based approach whether to break confidentiality and contact emergency services. These methods are in line with the best practice guidelines from the National Suicide Prevention Alliance.

Results In 2016/17, five issues required escalation, equivalent to $0.1 \%$ of posts. One of these individuals later confirmed they had got support from family members and the Samaritans.

Moderator Feedback 'This process makes me feel much more confident. I know that if I spot something worrisome, I don't need to panic - it's a simple process, and I won't be dealing with it on my own.'

Conclusion The safeguarding process appears effective, but we will be able to draw more conclusions in future, when there have been a greater number of issues escalated. We will review the process annually and review individual cases after three months.

\section{P-32 DEVELOPING AN INNOVATIVE HOSPICE APP}

Angela Halley, Megan Veronesi. Royal Trinity Hospice, London, UK

\subsection{6/bmispcare-2017-hospice.59}

Background Royal Trinity Hospice supports around 2500 patients a year, $80 \%$ of whom live in the community.
Feedback from patients and carers was that it was difficult to get to grips with changing symptoms and medications, and to understand the support available at the hospice, particularly in the early days after being referred.

Aims The Royal Trinity Hospice app is designed to provide patients and carers living at home with the support and information they need, in a timely and accessible way.

Methods The content of the app was developed through collaboration between a multi-disciplinary team of staff at the hospice incorporating feedback from patients and carers. The app includes:

- Easy to understand information on medications and common symptoms.

- Advance care planning prompts which can be shared via email with relatives or hospice staff.

- A function to manage attendance at hospice outpatients groups and contact key hospice teams directly.

- Bereavement content for carers.

Challenges during the development process included a lack of skills and experience in digital product development, capacity challenges around generating content and securing buy-in among hospice staff.

Results The app was launched on iTunes in April 2017 and was downloaded 83 times in the first two months. Patients commented that it confirmed that they were receiving the best possible care. Carers commented that the information on symptoms was good to refer back to. Both patients and carers felt it would be of most benefit when they were first referred to the hospice.

Conclusions The app is still in its pilot phase. A full evaluation will be undertaken to disseminate learning, to understand the full impact on patient and carer outcomes and to determine whether the app merits further investment. However initial findings would indicate there is scope for hospices to enhance face-to-face care with digital tools.

\section{P-33 E-LEARNING FOR THE VOLUNTEER WORKFORCE}

Sarah Owens. Wigan and Leigh Hospice, Wigan, UK

\subsection{6/bmjspcare-2017-hospice.60}

More than 125000 volunteers in the UK contribute to hospice care in a wide range of roles (Hospice UK, 2006). Wigan and Leigh Hospice has over 750 volunteers who give their time in the varying departments within the hospice on a daily basis. As an organisation we felt it vital that we provide them with the skills that they needed to best fulfil their role. Initially this training was delivered face-to-face in groups, covering specific areas including health and safety, infection control and safeguarding. Topics that the paid staff completed, as although in a volunteer role, they are in effect working for the organisation so it was important that they received very similar information. This was effective and they found it helpful, however, a number of volunteers are in employment or studying so often found it difficult to attend a face-to-face sessions. On review it was thought that a more flexible way of delivering the training would be via an e-learning package that the volunteers could access within the hospice or from home at a time to suit them. A volunteer e-learning module was put together and it is expected that volunteers complete this every two years. The module sits on our mandatory training system which also acts as a learning management system holding 